\title{
LEISHMANIOSE EM EQUINOS DO MUNICÍPIO DE ARAGUAÍNA, TOCANTINS: LEVANTAMENTO DA SOROPREVALÊNCIA ATRAVÉS DA REAÇÃO DE IMUNOFLUORESCÊNCIA INDIRETA (RIFI)
}

Leishmaniosis in horses of the municipality of Araguaina, Tocantins: prevalence study by indirect immunofluorescence test

Leishmaniasis in caballos del municipio de Araguaína, Tocantins: estúdio de la

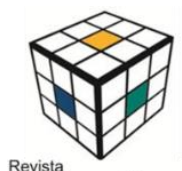
seroprevalencia por la reacción de inmunofluorescencia indirecta

\section{Letícia Oliveira Alencar ${ }^{* 1}$, Fabiana da Silva Carneiro Chagas ${ }^{* 2}$, Marco Augusto Giannoccaro da Silva ${ }^{* 3}$, Helcileia Dias Santos ${ }^{* 3}$}

${ }^{1}$ Curso de Medicina Veterinária, Universidade Federal do Tocantins, Araguaína, Brasil.

${ }^{2}$ Egresso do Programa de Pós-graduação em Sanidade Animal e Saúde Pública nos Trópicos, Universidade Federal do Tocantins, Araguaína, Brasil.

${ }^{3}$ Docente do Programa de Pós-graduação em Sanidade Animal e Saúde Pública nos Trópicos, Universidade Federal do Tocantins, Araguaína, Brasil.

*Correspondência: Programa de Pós-Graduação em Sanidade Animal e Saúde Pública nos Trópicos, Universidade Federal do Tocantins, BR 153, KM 112, Araguaína, Brasil. CEP:77.804-970. e-mail marcogiannoccaro@uft.edu.br.

\section{Artigo recebido em 13/03/2020 aprovado em 06/11/2020 publicado em 01/12/2020.}

\section{RESUMO}

Devido à sua localização geográfica no continente sul americano, o Brasil possui condições ambientais favoráveis que beneficiam a adaptação de várias espécies de artrópodes (DANTAS-TORRES, 2009) e, com isso, as populações humana e animal estão expostas a grande número de enfermidades por eles transmitidas, dentre as quais inclui-se a Leishmaniose Visceral (LV) (DE SOUZA et al., 2013), que representa um grave problema de saúde pública mundial e está em franco crescimento no Estado do Tocantins. Frente ao exposto, objetivou-se com o presente estudo pesquisar a presença de anticorpos anti-Leishmania infantum pela Reação de Imunofluorescência Indireta (RIFI) em equinos do município de Araguaína-TO. Utilizou-se 164 animais, dos quais 72 (43,90\%) foram reagentes na diluição 1:80. A presença de anticorpos anti-Leishmania infantum indica que o sistema imune do animal teve contato prévio com o protozoário, ou seja, evidencia a presença do parasita e a transmissão do mesmo na área de estudo (TRUPPEL et al., 2014). Com os resultados obtidos é possível inferir que os equinos de Araguaína estão em contato com os flebotomíneos transmissores da enfermidade e que o papel dos mesmos precisa ser elucidado, para que ações mais eficazes de prevenção da Leishmaniose Visceral possam ser instituídas na Cidade.

Palavras-chave: cavalo, Leishmania infantum, zoonose.

\section{ABSTRACT}

Brazil has favorable environmental conditions because of geographical location in the South American continent that benefit the adaptation of several species of arthropods (DANTAS-TORRES, 2009) and, with this, the human and animal populations are exposed to a large number of diseases transmitted by them, including Visceral Leishmaniasis (LV) (DE SOUZA et al., 2013), which represents a serious worldwide public health problem and is growing rapidly in the State of Tocantins. In view of the above, the objective of this study was to investigate the presence of antiLeishmania infantum antibodies by the Indirect Immunofluorescence test (IF) in horses in the municipality of Araguaina-TO. 164 animals were used, of which 72 (43.90\%) were reactive at 1:80 dilution. The presence of antiLeishmania infantum antibodies indicates that the animal's immune system had previous contact with the protozoan, 
that is, it evidences the presence of the parasite and its transmission in the study area (TRUPPEL et al., 2014). With the results obtained, it is possible to infer that the horses from Araguaina are in contact with the sandflies that transmit the disease and that their role needs to be elucidated, so that more effective actions to prevent Visceral Leishmaniasis can be instituted in the City.

Keywords: horse, Leishmania infantum, zoonosis.

\section{RESUMEN}

Debido a su ubicación geográfica en el continente sudamericano, Brasil tiene condiciones ambientales favorables que benefician la adaptación de varias especies de artrópodos (DANTAS-TORRES, 2009) y, con esto, las poblaciones humanas y animales están expuestas a una gran cantidad de enfermedades por ellos transmitidas, incluyendo la leishmaniasis visceral (LV) (DE SOUZA et al., 2013), que representa un grave problema de salud pública en todo el mundo y está creciendo rápidamente en el estado de Tocantins. Delante de lo expuesto, el objetivo de este estudio fue investigar la presencia de anticuerpos anti-Leishmania infantum por la reacción de inmunofluorescencia indirecta (RIFI) en caballos del municipio de Araguaína-TO. Se utilizaron 164 animales, de los cuales 72 (43.90\%) fueron reactivos en la dilución 1:80. La presencia de anticuerpos anti-Leishmania infantum indica que el sistema inmune del animal tuvo contacto previo con el protozoo, o sea, evidencia la presencia del parásito y su transmisión en el área de estudio (TRUPPEL et al., 2014). Con los resultados obtenidos, es posible inferir que los caballos de Araguaína atraen y están en contacto con los flebotomos que transmiten la enfermedad y que es necesario dilucidar el papel de los equinos, para que se puede imponer medidas más efectivas para prevenir la leishmaniasis visceral en la ciudad.

Descriptores: caballo, Leishmania infantum, zoonosis.

\section{INTRODUÇÃO}

Devido à sua localização geográfica no continente sul americano (zona tropical), o Brasil possui condições ambientais favoráveis que beneficiam a adaptação de várias espécies de artrópodes (DANTAS-TORRES, 2009). Consequentemente, as populações humana e animal estão expostas a um grande número de enfermidades por eles transmitidas, dentre as quais inclui-se a Leishmaniose Visceral (LV), que causa efeitos devastadores nestes seres (DE SOUZA et al., 2013). O vetor responsável por sua disseminação é a fêmea de dípteros da família Psychodidae, das espécies Lutzomyia longipalpis e Lutzomyia cruzi (ESCOBAR, 2015), conhecidos popularmente como mosquito-palha, birigui ou tatuquiras (COSTA, 2011).

A urbanização da enfermidade foi associada a adaptação do parasito a novos reservatórios, principalmente os domésticos (BARBOSA-SANTOS et al., 1994; FALQUETO et al., 1986, 1987; REITHINGER et al., 2007; SESSA et al., 1994), ao aumento da densidade populacional nos grandes centros, ao agravamento das desigualdades sociais e, à falta de saneamento básico e de moradias adequadas (GONTIJO e MELO, 2004; HARHAY et al., 2011).

No Estado do Tocantins, a construção da cidade de Palmas, o desenvolvimento de atividades ligadas à invasão das matas, a preservação de hábitos simples pela população como a criação de animais no quintal de casa, a presença frequente de animais vadios nas ruas das cidades e a falta de saneamento básico desencadearam alterações eco-epidemiológicas que culminaram na urbanização do vetor e na propagação da doença (CIMERMAN e CIMERMAN, 2003 in PARTATA, 2010).

Os cães, dentre os possíveis reservatórios urbanos, são considerados os principais do ponto de vista epidemiológico (CAMARGO-NEVES, 2004), além de serem a mais importante fonte de infecção para os flebotomíneos (SILVA, 2010). Porém, a eutanásia de animais positivos não tem surtido efeito no controle de novos casos (DE SOUZA et al., 2013), o que permite 
supor que outros animais como o gato e o cavalo, podem também exercer o papel de reservatórios (BARBOSA-SANTOS et al., 1994; UCHÔA et al., 2001; MADEIRA et al., 2003; JESUS et al., 2006), o que explicaria a ineficácia das medidas atuais de controle e demonstra a necessidade de novos estudos

Frente a isso, objetivou-se com a presente pesquisa verificar a ocorrência de infecção por Leishmania infantum em equinos do município de Araguaína-TO, empregando-se a Reação de Imunofluorescência Indireta (RIFI).

\section{MATERIAIS E MÉTODOS}

Utilizou-se na presente pesquisa 164 equinos, sendo 123 machos e 41 fêmeas, de diferentes raças e idades, criados na zona rural $(n=110)$ ou urbana $(n=54)$ da cidade de Araguaína-TO. Inicialmente, coletou-se amostras de animais que viviam em áreas onde a casuística de Leishmaniose Visceral Canina (LVC) era elevada e, posteriormente, para atingir a amostragem necessária, animais de outras propriedades também foram amostrados. Previamente a coleta, os equinos foram submetidos a exame físico geral seguindo protocolo sugerido por Speirs (1997), compondo o grupo experimental apenas os clinicamente saudáveis. Para realização deste trabalho obteve-se aprovação junto ao Comitê de Ética de Uso de Animais da UFT CEUA/UFT ( ${ }^{\circ}$ 23101.00-006990/2016-57).

As amostras de sangue foram coletadas por venopunção da jugular externa, utilizando-se sistema à vácuo e frascos sem anticoagulante. Imediatamente após a coleta, os frascos foram armazenados em caixa isotérmica contendo gelo reciclável até a chegada ao laboratório. Ato contínuo, as amostras foram centrifugadas para a obtenção dos soros, que foram então armazenados invidualmente em tubos do tipo eppendorf e congelados a $-20^{\circ} \mathrm{C}$ até o momento da análise.

Para a RIFI, o soro dos animais foi diluído inicialmente em 1:40 assim como o controle positivo e o negativo. Quando formas promastigotas fluorescentes foram visualizadas em uma amostra, esta foi considerada reagente e realizava-se nova diluição (1:80) e leitura. Os resultados expressos abaixo em frequência englobam apenas os reagentes nesta última diluição.

\section{RESULTADOS E DISCUSSÃO}

Anticorpos anti-Leishmania infantum foram detectados em 43,90\% (72/164) das amostras de soro equino avaliadas. Destas, 22,22\% (16/72) pertenciam a animais da zona urbana de Araguaína e 77,78\% (56/72) da zona rural.

$\mathrm{O}$ resultado indica que o sistema imune dos animais teve contato prévio com o protozoário, ou seja, evidencia a presença do parasita e a transmissão do mesmo na área de estudo (TRUPPEL et al., 2014). Tal fato corrobora com o postulado por Aguillar et al. (1989), Feitosa et al. (2012) e Kouam et al. (2010), de que os equinos são capazes de se infectar.

No entanto, o papel da espécie na transmissão das leishmanioses ainda é obscuro, pois eles podem tanto servir como fonte alimentar para os vetores devido à sua grande quantidade de sangue como, de fato, participar do ciclo epidemiológico da doença. De Colmenares et al. (1995) comprovaram que o flebótomo (Phlebótomus perniciosus) se alimenta do sangue equino e afirmaram não ser possível excluir os equinos da cadeia epidemiológica da $L$. infantum. Ainda, Bongiorno et al., (2003) asseguraram que o vetor não é espécie-específico e Fernández-Bellon et al., (2006) garantiram que em áreas endêmicas, como a cidade de Araguaína, onde os equinos coabitam com 
cães, estes estarão em exposição frequente ao agente e desenvolverão resposta imune humoral e celular, sem manifestar sinais da enfermidade.

\section{CONCLUSÃO}

Conclui-se que os equinos do município de Araguaína estão em contato com flebotomíneos transmissores da enfermidade; e, que outros testes precisam ser realizados para que o papel da espécie seja elucidado, tornando possível a instituição de ações mais eficazes para a prevenção da Leishmaniose Visceral.

\section{AGRADECIMENTOS}

O presente trabalho foi realizado com o apoio do Programa Nacional de Cooperação Acadêmica na Amazônia-PROCAD/Amazônia da Coordenação de Aperfeiçoamento de Pessoal de Nível SuperiorCAPES/Brasil. Ao Laboratório de Parasitologia Veterinária da UFT.

Todos os autores declararam não haver qualquer potencial conflito de interesses referente a este artigo.

\section{REFERÊNCIAS}

BARBOSA-SANTOS, E.G.O. et al. Leishmaniasis disseminated by Leishmania braziliensis in a mare (Equus cabalus): immunotheraphy and chemotherapy assays. Mem. Inst. Oswaldo Cruz, v. 89, p. 217-220, 1994.

BONGIORNO, G. et al. Host preferences of phlebotomine sand flies at a hypoendemic focus of canine leishmaniasis in central Italy. Acta Trop. 88, p. 109-116, 2003.

CAMARGO-NEVES, V.L.F. A leishmaniose visceral Americana no Estado de São Paulo: situação atual. Boletim Epidemiológico Paulista, v. 1, p. 1-4, 2004.
COSTA, C.H.N. How effective is dog culling in controlling zoonotic visceral leishamniasis? A critical evaluation of the science, politics and ethics behind this public health policy. Revista da Sociedade Brasileira de Medicina Tropical, v.44, n.2, p.232-242, 2011.

DANTAS-TORRES, F; Canine leishmaniosis in South America. Parasites \& Vectors. v.2 (Suppl I), 2009.

DE COLMENARES M. et al. Short report: detection of $72-75-\mathrm{kD}$ and $123-\mathrm{kD}$ fractions of Leishmania antigen in urine of patients with visceral leishmaniasis.

Am J Trop Med Hyg, v. 52: p. 427-8, 1995.

DE SOUZA, Y.C.P. Testes diagnósticos para leishmaniose visceral - atualidade e perspectivas.

Revista Científica Eletrônica de Medicina Veterinária. Garça, Editora FAEF, v. 21, 2013.

ESCOBAR, T.A. Investigação da presença de Leishmania sp. em equinos de zona urbana de Uruguaiana, RS. 2015. 60f. Mestrado (Ciência Animal), Universidade Federal do Pampa, Uruguaiana, 2015.

FALQUETO, A.; COURA, J.R.; BARROS, G.C.; et al. Participação do cão no ciclo de transmissão da Leishmaniose tegumentar no Município de Viana, Estado do Espírito Santo, Brasil. Mem. Inst. Oswaldo Cruz, v.81, n.1, p.155-163, 1986.

FALQUETO, A.; VAREJÃO, J.B.M.; SESSA, P.A. Cutaneous leishmaniasis in a horse (Equus cabalus) from endemic area in the state of Espírito Santo. Mem. Inst. Oswaldo Cruz, v.82, n.3, p.443, 1987.

FEITOSA, F.L.F. et al. Nota prévia: Estudo soroepidemiológico de leishmaniose em equinos na região de Araçatuba-SP, Brasil, área endêmica para leishmaniose visceral. Brazilian Journal of Veterinary Research and Animal Science, São Paulo, v. 49, n. 6, p. 500-502, 2012.

FERNÁNDEZ-BELLON, H. et al. Immune response to Leishmania infantum in healthy horses in Spain. Veterinary Pathology, v. 135, p. 181-185, 2006.

GONTIJO, C.M.F.; MELO, M.N. Visceral Leishmaniasis in Brazil: current status, challenges and 
prospects. Rev. Bras. Epidemiol. v.7, p. 338-349, 2004.

HARHAY, M.O.; OLLIARO, P.L.; COSTA, D.L.; COSTA, C.H. Urban parasitology: visceral leishmaniasis in Brazil. Trends Parasitol., v.27, n._, p.403- 409, 2011.

JESUS, J.R. et al. Avaliação sorológica de anticorpos para Leishmania spp. na população canina em região de foco de leishmaniose tegumentar americana na Lomba do Pinheiro, Porto Alegre, Rio Grande Do Sul, Brasil. Parasitol. Latinoam., v.61, n._, p.121-125, 2006.

KOUAM, M. K. et al. A seroepidemiological study of exposure to Toxoplasma, Leishmania, Echinococcus and Trichinella in equids in Greece and analysis of risk factors. Veterinary Parasitology, v. 170, n. 1, p. 170$175,2010$.

MADEIRA, M.F. et al. Leishmania (Viannia) braziliensis em cães naturalmente infectados. Rev. Soc. Bras. Med. Trop., v.36, n.5, p.551-555, 2003.

PARTATA, A.K. Epidemiologia da leishmaniose visceral humana em Araguaína (TO) e o diagnóstico sorológico da doença. 2010. 75f. Tese (Doutorado em Ciências). Instituto de Pesquisas Energéticas e Nucleares, Universidade de São Paulo, São Paulo, 2010.
REITHINGER. R.; DUJARDIN, J.C. Molecular Diagnosis of Leishmaniasis: Current Status and Future Applications. J. Clin. Microbiol., v. 45, n. 1, p. 21-25, 2007.

SESSA, P.A.; FALQUETO, A.; VAREJÃO, J.B.M. Tentativa de controle da leishmaniose tegumentar americana por meio do tratamento dos cães doentes. Ca. Saúde Public., v.10, n.4, p.457-463, 1994.

SILVA, D.A. Avaliação clínica e laboratorial de cães sororreatores para Leishmania provenientes de inquérito sorológico realizado no município do Rio de Janeiro. 2010. 54f. Dissertação (Mestrado em Pesquisa Clínica em Doenças Infecciosas) - Instituto de Pesquisa Clínica Evandro Chagas, Fundação Oswaldo Cruz, Rio de Janeiro.

SPEIRS, V.C. Clinical examination of horses. Philadelphia: Saunders, 1997. 349p.

TRUPPEL, J.H. et al. Can equids be a reservoir of 287 Leishmania braziliensis in Endemic Areas. Plos One, v. 9, n. 4, p.1-7, 2014.

UCHÔA, C.M.A. et al. Aspectos sorológicos e epidemiológicos da leishmaniose tegumentar americana canina em Maricá, Rio de Janeiro, Brasil. Rev. Soc. Bras. Med. Trop., v. 34, p.563-568. 